\title{
Associations between daily-living physical activity and laboratory-based assessments of motor severity in patients with falls and Parkinson's disease
}

\author{
Irina Galperin ${ }^{\mathrm{a}}$, Inbar Hillel ${ }^{\mathrm{a}}$, Silvia Del Din ${ }^{\mathrm{b}}$, Esther M.J. Bekkers ${ }^{\mathrm{c}}$, Alice Nieuwboer ${ }^{\mathrm{c}}$, \\ Giovanni Abbruzzese $^{\mathrm{e}, \mathrm{f}}$, Laura Avanzino ${ }^{\mathrm{f}, \mathrm{g}}$, Freek Nieuwhof ${ }^{\mathrm{d}, \mathrm{h}}$, Bastiaan R. Bloem ${ }^{\mathrm{d}}$, \\ Lynn Rochester ${ }^{\mathrm{b}, \mathrm{i}}$, Ugo Della Croce ${ }^{\mathrm{j}, \mathrm{k}}$, Andrea Cereatti ${ }^{\mathrm{j}, \mathrm{k}, \mathrm{l}}$, Nir Giladi ${ }^{\mathrm{a}, \mathrm{n}, \mathrm{o}}$, Anat Mirelman ${ }^{\mathrm{a}, \mathrm{n}, \mathrm{o}}$, \\ Jeffrey M. Hausdorff ${ }^{a, m, n, p, *}$
}

${ }^{\text {a }}$ Center for the study of Movement, Cognition and Mobility, Neurological Institute, Tel Aviv Sourasky Medical Center, Israel

${ }^{\mathrm{b}}$ Institute of Neuroscience, Newcastle University Institute for Ageing, Clinical Ageing Research Unit, Campus for Ageing and Vitality, Newcastle University, Newcastle upon

Tyne, UK

${ }^{\mathrm{c}}$ Neuromotor Rehabilitation Research Group, Department of Rehabilitation Sciences, KU Leuven, Belgium

${ }^{\mathrm{d}}$ Donders Institute for Brain, Cognition and Behaviour; Radboudumc, Department of Neurology, Nijmegen, the Netherlands

${ }^{\mathrm{e}}$ Department of Neuroscience, Rehabilitation, Ophthalmology, Genetics and Maternal Child Health, University of Genova, Italy

${ }^{\mathrm{f}}$ IRCCS San Martino Teaching Hospital, Genoa, Italy

${ }^{\mathrm{g}}$ Department of Experimental Medicine, Section of Human Physiology, University of Genova, Italy

${ }^{\mathrm{h}}$ Radboud university Medical Center, Departments of Geriatric Medicine, Neurology and Parkinson's Disease Center Nijmegen (ParC), Nijmegen, the Netherlands

i The Newcastle upon Tyne Hospitals NHS Foundation Trust, UK

${ }^{\mathrm{j}}$ Department of Biomedical Sciences, Bioengineering unit, University of Sassari, Sassari (SS), Italy

${ }^{\mathrm{k}}$ Interuniversity Centre of Bioengineering of the Human Neuromusculoskeletal System, Sassari (SS), Italy

${ }^{1}$ Department of Electronics and Telecommunications, Politecnico di Torino, Torino (TO), Italy

${ }^{\mathrm{m}}$ Department of Physical Therapy, Sackler Faculty of Medicine, Israel

${ }^{\mathrm{n}}$ Sagol School of Neuroscience, Tel Aviv University, Israel

${ }^{\circ}$ Department of Neurology and Neurosurgery, Sackler School of Medicine, Tel Aviv University, Israel

${ }^{\mathrm{p}}$ Rush Alzheimer's Disease Center and Department of Orthopaedic Surgery, Rush University Medical Center, Chicago, USA

\section{A R T I C L E I N F O}

\section{Keywords:}

Parkinson's disease

Wearable device

Accelerometers

Inertial measurement units

Digital health

Daily-living activity

\begin{abstract}
A B S T R A C T
Introduction: Recent work suggests that wearables can augment conventional measures of Parkinson's disease (PD). We evaluated the relationship between conventional measures of disease and motor severity (e.g., MDSUPDRS part III), laboratory-based measures of gait and balance, and daily-living physical activity measures in patients with PD.

Methods: Data from 125 patients (age: $71.7 \pm 6.5$ years, Hoehn and Yahr: 1-3, 60.5\% men) were analyzed. The MDS-UPDRS-part III was used as the gold standard of motor symptom severity. Gait and balance were quantified in the laboratory. Daily-living gait and physical activity metrics were extracted from an accelerometer worn on the lower back for 7 days.

Results: In multivariate analyses, daily-living physical activity and gait metrics, laboratory-based balance, demographics and subject characteristics together explained $46 \%$ of the variance in MDS-UPDRS-part III scores. Daily-living measures accounted for $62 \%$ of the explained variance, laboratory measures $30 \%$, and demographics and subject characteristics $7 \%$ of the explained variance. Conversely, demographics and subject characteristics, laboratory-based measures of gait symmetry, and motor symptom severity together explained less than $30 \%$ of the variance in total daily-living physical activity. MDS-UPDRS-part III scores accounted for $13 \%$ of the explained variance, i.e., $<4 \%$ of all the variance in total daily-living activity.

Conclusions: Our findings suggest that conventional measures of motor symptom severity do not strongly reflect daily-living activity and that daily-living measures apparently provide important information that is not captured in a conventional one-time, laboratory assessment of gait, balance or the MDS-UPDRS. To provide a more complete evaluation, wearable devices should be considered.
\end{abstract}

\footnotetext{
${ }^{*}$ Corresponding author. Center for the Study of Movement, Cognition, and Mobility, Department of Neurology, Tel Aviv Sourasky Medical Center, Tel Aviv, Israel. E-mail address: jhausdor@tlvmc.gov.il (J.M. Hausdorff).
} 


\section{Introduction}

Difficulties in gait, balance, and mobility are major contributors to disability, diminished quality of life and fall risk in patients with Parkinson disease's (PD). In the past, these symptoms have generally been quantified in the laboratory and in clinical settings. Emerging evidence suggests that there are key differences between gait measured in the clinic or laboratory setting versus measures assessed during dailyliving [1-4]. Furthermore, measures based on community ambulation apparently may help to predict important outcomes such as fall risk $[5,6]$ and quality of life [7]. These findings support the idea that the assessment of mobility during daily-living provides information that is complementary to more conventional clinic and laboratory assessments of gait and motor function.

Several recent studies used wearable sensors to characterize and quantify daily-living physical activity among patients with PD [8-13]. Not surprisingly, the results of these studies suggest that everyday mobility differs in people with PD, as compared with age-matched controls, and that daily-living activity measures have utility in assessing and tracking PD by reflecting the subject's movement at home and in the community. Since activity and every-day function may be influenced by factors such as cognitive function, affect, environment, and social interactions, measures of daily-living may capture features that are not reflected in a single test in a laboratory or clinical setting. However, it has not been fully explored if and how the quality and quantity of daily-life physical activity characteristics relate to the conventional assessment of motor symptom severity in PD.

The assessment of disease severity is routinely conducted in the clinic using the Unified Parkinson's Disease Rating Scale (MDS-UPDRS) [14]. The motor section subscale of the MDS-UPDRS (part III) evaluates motor symptom severity and is often used as the primary outcome for clinical studies in PD. It is widely accepted that gait performance, when measured in the clinic, correlates with disease severity [15]. It is also well-established that daily-living physical activity is related to morbidity and mortality and that it may positively modify disease severity, improving a wide range of global and specific motor and non-motor symptom in PD [16-18]. Nonetheless, the relationships between the severity of the motor symptoms in PD, gait, and balance, as measured during a one-time visit to the clinic, and measures based on daily-living mobility are not clearly understood.

One could speculate that people with more severe motor symptoms are less active. However, since activity may be affected by more than just motor symptoms and abilities, this relationship may be more complex. Several studies found associations between some items of the UPDRS-part III with sensor-derived gait acceleration in PD patients [19-21]. Yet, those studies did not explore the motor severity-dailyliving physical activity relationship considering gait and balance assessed in the laboratory setting. Therefore, in the present analysis, we evaluated the relationship between PD motor symptom severity and metrics based on the laboratory-based assessment of mobility, on the one hand, and the daily-living assessment of mobility, on the other. In addition, since total daily-living physical activity is associated with many positive and negative health care outcomes (e.g., dementia, mortality) and since its benefits are well established [22,23], we investigated the relationship between total daily-living physical activity (based on objective ambulatory monitoring), clinic-based assessment of disease severity (MDS-UPDRS part I-III) and clinic-based metrics of mobility (gait and balance).

\section{Methods}

\subsection{Participants}

The present analysis is based on the baseline assessment of subjects who participated in a randomized controlled trial designed to reduce fall rates in older adults, as detailed previously [24]. Briefly, the study was conducted at 5 clinical centers (Belgium, Israel, Italy, the Netherlands, and United Kingdom). The inclusion criteria for the present analyses were: (a) People diagnosed with PD according to the UK Brain Bank criteria by a movement disorders specialist (b) age 60-90 years, (c) Hoehn and Yahr stage I-III, (d) taking anti-parkinsonian medications and with stable prescriptions at least for the past month, (e) had at least two falls in the 6 months prior to assessment, and (f) able to walk at least 5 min without assistance and (f) physical activity recording times greater than 3 days. Subjects were excluded if they had other significant comorbidities, clinical diagnosis of dementia or severe cognitive impairment (Mini Mental State Exam score, MMSE < 21). The study was approved by each clinical site's ethics committee. All participants provided informed written consent prior to testing.

\subsection{Assessment of demographics and other subject characteristics}

Age, gender, and other subject characteristics were collected. Motor symptom severity was assessed using the motor part of the MDSUPDRS, i.e., part III [14] in a self-reported ON stage. Parts I and II of the MDS-UPDRS evaluated motor and non-motor experiences of dailyliving based on-self-report.

\subsection{Laboratory-based assessment of mobility}

The participants walked back and forth in a well-lit corridor of $15 \mathrm{~m}$ for $1 \mathrm{~min}$ at a preferred, usual walking pace. Gait measures (e.g., speed, step length, and stride time variability) were collected using a Zeno instrumented walkway and PKMAS software, (Havertown, PA, USA) [25] and an inertial measurement unit placed on the lower back (Opal, APDM, Portland, OR, USA). Only straight-line walks, defined as sagittal progression walking, were analyzed. To compare laboratory to dailyliving gait, only acceleration-based features were calculated from the inertial measurement unit. To further assess balance, the Mini-Balance Evaluation Systems Test (Mini-BESTest) and the Four Square Step Test were used. Endurance was evaluated using the Two Minute Walk Test by measuring the total distance covered [26].

\subsection{Daily-living assessment of physical activity}

At the end of the laboratory testing session, a small, light-weight, water-proof, tri-axial accelerometer (Axivity AX3, York, UK; $23.0 \times 32.5$ $\times 7.6 \mathrm{~mm}$; weight: $11 \mathrm{~g} ; 100 \mathrm{~Hz}$ sampling rate) was placed on each subject's lower back at the level of the fifth lumbar vertebrae, as previously described [11]. The device was attached with a hydrogel adhesive and covered with a Hypafix bandage. Participants were asked to wear the device continuously for one week and to continue their daily activities as usual. Upon completion of the one-week recording, participants removed the device and sent it back to the local clinical site.

\subsection{Daily-living activity metrics}

As previously described [9], an algorithm automatically identified the different activities (walking, lying, standing, and sitting) and each bout of walking throughout the week-long recording and then extracted measures that reflect the quantity and quality of walking. To focus on steady-state walking and to compare in-lab walking with community ambulation, we evaluated walking bouts that were at least $60 \mathrm{~s}$ long [9]. However, when focusing on gait quantity, we used walking bouts of all lengths. The extracted measures are defined in Supplementary Material Table 1; these include measures of gait quantity (e.g., number of steps, number of walking bouts) and gait quality. Gait quality measures included those that reflect pace (e.g., step length), gait symmetry (e.g.,.step regularity) gait variability (e.g., the amplitude of dominant frequency) and variability across walking bouts (e.g., SD of the peaks amplitude CV). To describe the overall level and distribution of physical activity intensity, we averaged the vector magnitude value over 15-s 
epoch, similar to Doherty et al. [27]. Then, we generated the signal vector magnitude, SVM measurement, an empirical cumulative distribution function from all available 15-s epochs (for further information on how SVM reflects daily-living physical activity, see Supplementary Material Methods). Data was included in the analysis if the recording was longer than three days.

\subsection{Statistical analyses}

A series of multivariable linear regression analyses were performed to identify independent predictors associated with the two dependent measures of interest: 1) motor symptom severity as expressed by the MDSUPDRS-part III; and, in separate analyses, 2) total daily-living physical activity level, as expressed by the SVM of the acceleration signal, summed over the week. Normality of data was assessed using Shapiro-Wilk tests. To avoid colinearity, we first examined the relationships among metrics within daily-living and within laboratory subcategories using Pearson's correlations. If two metrics were strongly correlated with one another $(r>0.7)$, only the one most strongly associated with the dependent variable (e.g., MDS-UPDRS-part III) was retained. Subsequently, we carried out a series of backward regression models to identify the relationship between the dependent outcome and independent factors associated with the dependent variable by examining each family of measures first (e.g., balance and functional tests, gait quantity, gait pace, gait symmetry, gait variability, day and night activity, and variability across walking bouts, demographics and subject's characteristics and MDS-UPDRS I-III) and then generating a single, parsimonious model. The process is summarized in Supplementary Material Fig. 1. A variable was entered into the model if the significance level of its $F$ value of the ANOVA was less than 0.05 and was removed if the significance level is greater than 0.10 . All analyses were adjusted for age, sex and disease duration. All of the independent predictors that were identified within each set were then entered into another backward regression model to identify the laboratory and daily-living predictors. Finally, the predictors that remained in those models were entered into a final regression model. We report the beta and p-values for the predictors. SPSS version 24 was used for the statistical analyses.

\section{Results}

The subjects had moderate disease severity (Hoehn \& Yahr 1-3) and were multiple fallers (at least two falls in 6 months prior to assessment). They were generally well-educated, did not have major cognitive impairments, and had approximately 10 years of motor symptoms (Table 1). The univariate relationships between the MDS-UPDRS-parts I-III (and the total score), two selected laboratory-based gait and balance measures, and total daily-living physical activity are summarized in Fig. 1. Total daily-living physical activity was not strongly correlated with the scores on any of the MDS-UPDRS tests or with the lab-based assessment of balance or gait. Gait speed and MiniBest scores were moderately correlated with MDS-UPDRS-part III scores.

Table 1

Participant characteristics (entries are mean \pm SD, median (range) or \%).

\begin{tabular}{ll}
\hline Variable & PD $(\mathrm{n}=125)$ \\
\hline Age (yrs) & $71.49 \pm 6.38$ \\
Gender $(\%$ men) & $60.52 \%$ \\
Education (yrs) & $13.34 \pm 4.39$ \\
Body-mass-index (kg/m2) & $25.91 \pm 3.67$ \\
Disease Duration (yrs) & $9.13 \pm 6.42$ \\
Levodopa Equivalent Daily Dose (mg/kg) & $970 \pm 608$ \\
Hoehn and Yahr Stage & $2.5(1-3)$ \\
Montreal Cognitive Assessment & $23.87 \pm 4.21$ \\
Mini Mental State Examination & $28.02 \pm 1.69$ \\
UPDRS Total score & $63.47 \pm 21.53$ \\
UPDRS Part III (Motor) & $30.43 \pm 13.04$ \\
Falls in 6 month prior to assessment & $3(2-50)$
\end{tabular}

The multivariate associations between each laboratory and dailyliving family of metrics and PD motor symptom, i.e., MDS-UPDRS-part III, are summarized in Supplementary Material Table 2. Age, sex and disease duration explained 6.0\%, laboratory-based measures of gait and balance explained $27.1 \%$, and daily-living measures explained $37.8 \%$ of the variance of the MDS-UPDRS-part III scores. When considered together in a final block-wise regression model, the predictors explained $46.2 \%$ of the variance in the MDS-UPDRS-part III (see Table 2a). As shown in Fig. 2a, daily-living measures accounted for $62.0 \%$ of the explained variance, laboratory measures for $30.1 \%$, demographics and subject characteristics for $7.7 \%$ of the explained variance of PD motor symptom severity as assessed in the clinic using the MDS-UPDRS-part III.

Examining these relationships from a different perspective, Supplementary Material Table 3 summarizes the contribution of demographics and subject characteristics, MDS-UPDRS (parts I-III) scores and laboratory gait and balance measures to the variance in total dailyliving physical activity (i.e., SVM). Age, sex and disease duration explained $12.4 \%$ of the variance, as shown in Model A. Since women had significantly fewer years of disease duration and significantly higher SVM, we added a disease duration and sex interaction term to the model. As shown in Model B, MDS-UPDRS-part III had the strongest association with total daily-living physical activity among the three UPDRS tests, predicting $15.8 \%$ of its variance when considered together with demographics and subject characteristics. However, the MDSUPDRS-part III alone accounted for only 5.8\%. Laboratory-based balance and gait measures explained $27.6 \%$ of the SVM variance, as summarized in Model C.

When considered together in a final block-wise regression model, $27.1 \%$ of the variance of total daily-living physical activity was explained (see Table 2b). Laboratory gait symmetry measures accounted for $44.0 \%$, demographics and subject characteristics for $42.7 \%$, and the MDS-UPDRS-part III for $13.3 \%$ of the explained variance of the total daily-living physical activity (see Fig. 2b); in other words, the MDSUPDRS explained less than $4 \%(0.13 \times 0.27)$ all of the variance in total daily-living physical activity. Scores on the MDS-UPDRS parts I and II were not independently associated with total daily-living physical activity.

\section{Discussion}

The results of this cross-sectional analysis indicate that daily-living physical activity, laboratory-based measures of dynamic balance, demographics and subject characteristics are related to PD motor symptom severity, as expected, and that they explain almost $50 \%$ of the variance of motor symptom severity (recall Fig. 2a). On the flip side, demographics and subject characteristics, laboratory-based gait and balance tests, and disease severity (i.e., MDS-UPDRS I-III) explained less than a third of the total daily-living physical activity (recall Fig. 2b). Taken together, these findings suggest that home-based, 7 days continuous daily-living-based measures of mobility and function are related to traditional measures of disease severity, specifically MDSUPDRS part III. In addition, daily-living measures also apparently provide additional information that is not strongly reflected by conventional standardized, one-time measures conducted in the clinic (e.g., MDS-UPDRS I-III, Mini-BESTest).

\subsection{The contribution of laboratory-based and daily-living physical activity measurements to the variance of PD motor symptom severity}

Our results suggest that daily-living mobility monitoring adds considerable explanatory value to the severity of motor symptoms in PD. We found that daily-living activity measures independently explain $37.8 \%$ of the variance of the severity of motor symptoms, as reflected by the MDS-UPDRS-part III (see Supplementary Material Table 2). This relatively high value is somewhat surprising when considering that the 


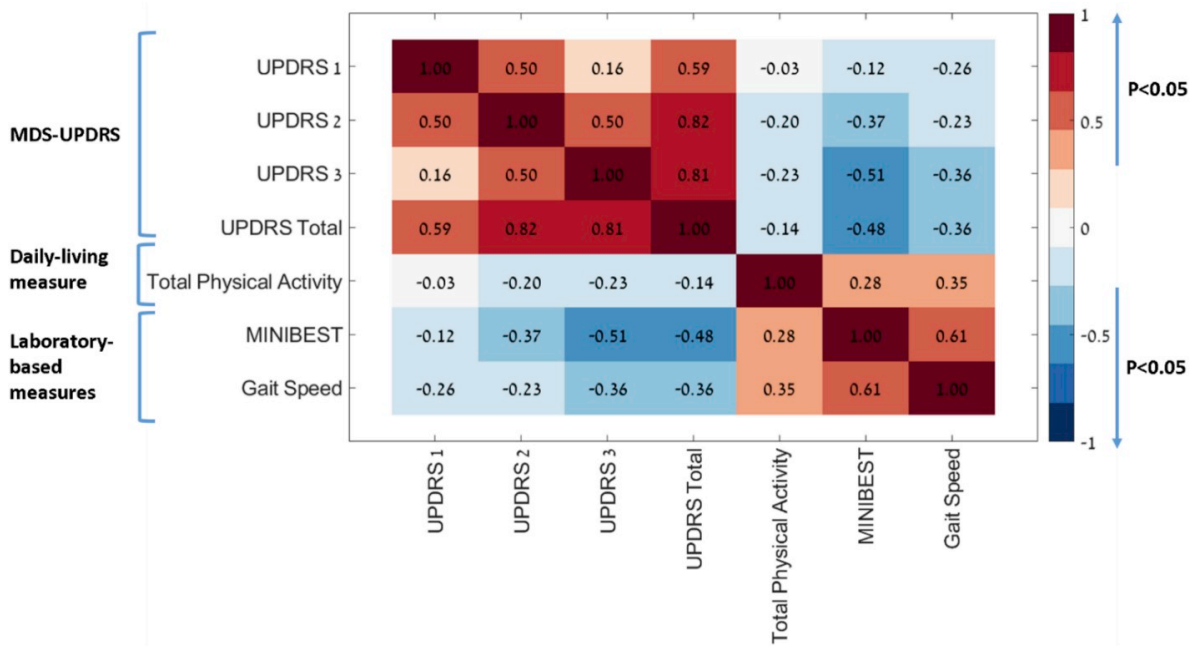

Fig. 1. Heat map showing the univariate Pearson correlation coefficients between MDS-UPDRS, daily living activity, specifically, total daily-living physical activity (SVM), and lab-based measures of gait and balance. Darker pixels reflect higher correlation values. Note that MDS-UPDRS parts I-III and total scores are only moderately correlated to total dailyliving physical activity. Gait speed and the MiniBest were chosen as representatives of lab-based representatives because of their widespread use and because of their relatively strong association with MDS-UPDRS 3. Similar results were obtained using Spearman's correlation instead of Pearson's.
Table 2a

Multivariable model of joint contributions of lab-based and daily-living measures to the variance in motor symptom severity, as evaluated by the MDSUPDRS part III.

\begin{tabular}{|c|c|c|c|}
\hline & $\begin{array}{l}\mathrm{R}^{2} \text { within } \\
\text { block }\end{array}$ & $\mathrm{R}^{2}$ Change & Total $\mathrm{R}^{2}$ \\
\hline $\begin{array}{l}\text { Demographics and subject characteristics } \\
\text { Age }(\beta=0.193) \\
\text { Disease Duration }(\beta=0.177)\end{array}$ & 0.061 & 0.061 & 0.462 \\
\hline $\begin{array}{l}\text { Laboratory Gait and Balance Measures** } \\
\text { Age }(\beta=0.017) \\
\text { Disease Duration }(\beta=0.081) \\
\text { Mini-BESTest }(\beta=-0.506)\end{array}$ & 0.281 & 0.220 & \\
\hline $\begin{array}{l}\text { Daily-Living Measures ** } \\
\text { Age }(\beta=-0.051) \\
\text { Disease Duration }(\beta=0.075) \\
\text { Mini-BESTest }(\beta=-0.490) \\
\text { Gait Quantity: number of walking bouts } \\
\quad \text { above } 120 \mathrm{~s} \text { long }(\beta=0.348) \text { number of } \\
\quad \text { walking bouts } 5-10 \mathrm{~s} \text { long }(\beta=-0.186) \\
\text { Gait Variability: } \\
\quad \text { ampML }(\beta=0.256) \\
\quad \text { frqML }(\beta=0.217)\end{array}$ & 0.462 & 0.182 & \\
\hline
\end{tabular}

$* *$ this block of analyses builds on the previous block. Initial steps leading to these results are shown in a supplementary material table.
Table 2b

Multivariable model of joint contributions of lab-based measures and motor symptom severity to the variance in total daily-living physical activity, as measured by the SVM.

\begin{tabular}{|c|c|c|c|}
\hline & $\begin{array}{l}\mathrm{R}^{2} \text { within } \\
\text { block }\end{array}$ & $\mathrm{R}^{2}$ change & Total $\mathrm{R}^{2}$ \\
\hline $\begin{array}{l}\text { Demographics and subject characteristics } \\
\text { Age }(\beta=-0.187) \\
\text { Sex }(\beta=-0.004) \\
\text { Disease Duration }(\beta=-0.178) *\end{array}$ & 0.113 & 0.113 & 0.271 \\
\hline $\begin{array}{l}\text { Motor Symptom Severity } * * \\
\text { Age }(\beta=-0.139) \\
\text { Sex }(\beta=0.001) \\
\text { Disease Duration }(\beta=-0.152) * \\
\text { MDS-UPDRS-part III }(\beta=-0.214)\end{array}$ & 0.155 & 0.042 & \\
\hline $\begin{array}{l}\text { Laboratory Gait and Balance Measures } * * \\
\text { Age }(\beta=-0.127) \operatorname{Sex}(\beta=-0.066) \text { Disease } \\
\quad \text { Duration }(\beta=-0.237) * \\
\text { MDS-UPDRS-part III }(\beta=-0.134) \\
\text { Gait Symmetry: } \\
\quad \text { StpRegV }(\beta=0.268) \\
\quad \text { HRv }(\beta=-0.174)\end{array}$ & 0.270 & 0.116 & \\
\hline
\end{tabular}

* Adjusted to sex-disease duration interaction.

** This block of analyses builds on the previous block; initial steps leading to these results are shown in a supplementary material table. a)

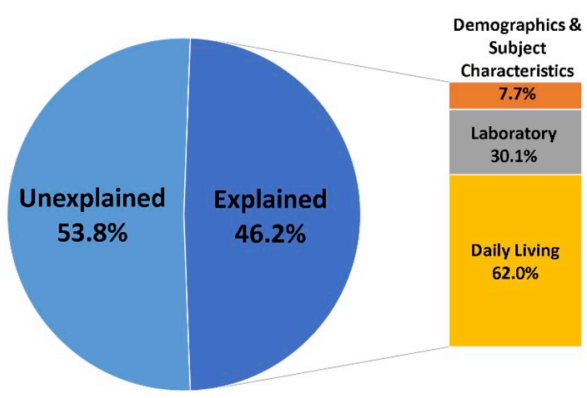

b)

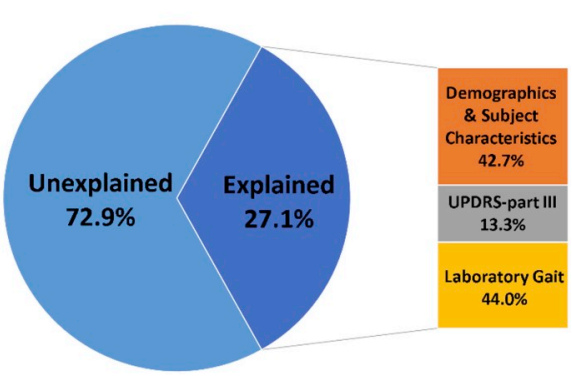

Fig. 2. Variance of the two dependent outcome measures. a) Variance in MDS-UPDRS-part III as explained by laboratory and daily-living metrics. Dailyliving measures accounted for $62.0 \%$, laboratory measures for $30.1 \%$ demographics and subject characteristics for $7.7 \%$ of the explained variance in the MDS-UPDRS part III: motor part of the Unified Parkinson's Disease Rating Scale; b) Variance in total physical daily-living physical activity, SVM, as explained by subject demographics, the MDS-UPDRS, and laboratory measures of gait and balance. Demographics and subject characteristics accounted for $42.7 \%$, MDS-UPDRS-part III $13.3 \%$ and laboratory gait measures for $44.0 \%$ proportion of the explained variance. Note that MDS-UPDRS parts I and II were included as potential predictors, but they were not significant independent predictors and hence were not included in the final model. (MDS-UPDRS part III: motor part of the Unified Parkinson's Disease Rating Scale). 
MDS-UPDRS-part III score is composed of items that evaluate a wide range of PD non-gait related symptoms including tremor, speech, facial expressions, rigidity, and bradykinesia. The MDS-UPDRS-part III items related to gait, balance and lower extremity movements account for approximately one-third of the total possible score. This demonstrates a noticeable advantage of assessing PD mobility using objective tools of home-based, continuous daily function when compared to laboratorybased assessment (mobility capacity) which may be influenced by the clinician's subjectivity and the patient's extra effort during a short term examination. Supported by other recent studies [19,28], our findings demonstrate the importance of the emerging approach of assessing PD motor disability in daily-living conditions based on continuous recordings, in addition to a conventional one-time, laboratory assessment. Our analysis revealed important associations between specific measures of daily-living activity (daily-living gait variability and the number of longer and shorter walking bouts) and severity of the motor symptoms in PD (recall Supplementary Material Table 2). Interestingly, the participants mainly used short 5-10 s walking bouts, whereas long bouts ( $>120 \mathrm{~s}$ ) were rare (recall Supplementary Material Table 1). A possible explanation for these findings may be that daily-living activities, mostly at home, were composed of a large number of short walking bouts. Perhaps, people with PD with worst disease severity prefer to get up for a "good reason" twice a day (the mean of large bouts in our analysis, recall Supplementary Material Table 1) instead of making more frequent short movements. Conceivably, improving strength and fitness in treatment sessions along with balance training may lengthen PD patients' walking bouts might, positively influence their motor disability and even reduce future fall risk.

4.2. The contribution of PD motor symptom severity and other laboratorybased assessment of mobility to the variance of total daily-living physical activity

When trying to explain total daily-living activity, all laboratory tests together (including gait, balance and the severity of motor symptom) accounted for less than $30 \%$ of its variance (recall Table $2 \mathrm{~b}$ ). These findings, supported in part by previous studies [4,28], are likely related to the influence of environmental conditions, motor and non-motor fluctuations, comorbidities and other putative mediators of daily-living physical activity that cannot be accounted for by one-time laboratory or clinic testing. The best predictors of total daily-living activity were demographics and subject characteristics (which were retained in all models), followed by gait symmetry measured in the laboratory (see Table 2b). Unexpectedly, balance tests, walking speed and step length measured in the lab did not contribute to the variance of the total dailyliving physical activity, nor did cognitive function (as represented by the Montreal Cognitive Assessment). Somewhat counter-intuitively, we found that only the MDS-UPDRS-part III score remained as a predictor (explaining approximately $16 \%$ of the variance in SVM, together with age, sex and disease duration), while the other two parts of the MDSUPDRS (ADL and non-motor symptoms) did not remain as predictors (see Supplementary Material Table 3). Yet, our results seem to be consistent with other research which found that higher age, gender and greater severity of motor symptoms are associated with less time spent walking [21] and total energy expenditure (kcal/day) [16]. As previously demonstrated, greater total daily-living physical activity is known to protect against a range of diseases and negative outcomes $[22,23]$ and is negatively associated with mortality in old age [29]. Moreover, recent studies showed that physical activity plays a role in improving a multitude of global and specific motor and non-motor symptom in PD patients $[17,18]$. Thus, in PD, daily physical activity may be considered as a form of non-pharmacological therapy [17]. Finally, the role of daily-living physical activity as an important predictor of many adverse health outcomes suggests that one needs to measure it to gain a more complete estimate of the impact of disease and treatments [30].
In conclusion, to the best of our knowledge, this is the first systematic examination of the relationship between daily-living physical activity and motor symptom severity in patients with PD. On the one hand, our findings revealed that standardized measures such as clinicbased MDS-UPDRS-part III along with other laboratory gait measures are relatively weak predictors of how patients actually function at home and in the community (i.e., outside of the clinic) (recall Table 2b and Figs. 1 and 2). On the other hand, specific daily-living activity measures apparently are relatively strong predictors of motor symptom severity (recall Table 2a and Fig. 2a). These findings emphasize the difference between clinical or laboratory testing and real-life activity of patients. Thus, the present results underscore the importance of monitoring daily-living activity for understanding disability and disease progression as well as potentially monitoring the effects of interventions and treatments.

\subsection{Limitations and future work}

The present work has several limitations. The cross-sectional nature of this analysis limits our ability to identify cause and effect and changes over time. The participants who were analyzed here were all, by definition, fallers. In the future, it will be important to see if similar associations are observed among PD subjects who are not fallers and among less severely impaired patients. Potential new metrics resulting from more advanced analytical approaches (and additional sensing technologies such as gyroscopes) could provide even greater input into the functional motor performance of patients during daily-living. Furthermore, it is not possible to fully separate passive and active acceleration from the measurement of a tri-axial accelerometer placed on the lower back alone. Nonetheless, the present findings suggest that measuring daily-living physical activity has strong potential to more fully and optimally assess patients with PD and to explore functional decline and changes over time in response to therapeutic interventions and potential deterioration. Prospective studies are needed to further evaluate the degree to which long-term, 24/7 monitoring of gait and physical activity adds new levels of granularity and additional relevant information, above and beyond more conventional, one-time assessments.

\section{Disclosures}

Lynn Rochester reports grants from Medical Research Council, grants from EU, grants from NIHR, grants from Wellcome, grants from EPSRC, grants from Parkinson's UK, grants from Stroke Association in the last 36 months outside the submitted work.

Bastiaan R. Bloem has declared that he received consultant services from Zambon and UCB. His grants/research support are: Netherlands Organisation for Scientific Research, Princess Beatrix Foundation, Michael J Fox Foundation, Parkinson Vereniging, National Parkinson Foundation, Hersenstichting Nederland, UCB, and AbbVie. He has received fees for speaking at conferences from AbbVie, Zambon, Biall.

Giovanni Abbruzzese received honoraria from Zambon (Italy) for Advisory Board participation.

Esther M.J. Bekkers and Alice Nieuwboer confirmed that there are no known conflicts of interest associated with this publication and there has been no significant financial support for this work that could have influenced its outcome. They acknowledge for partial funding of this project through a grant from Research Foundation Flanders (FWO) [grant number G.0867.15].

Silvia Del Din, Laura Avanzino, Freek Nieuwhof, Inbar Hillel and Ira Galperin declared they have no conflicts of interest associated with this publication and there has been no significant financial support for this work that could have influenced its outcome.

Nir Giladi reports that he is a consultant for: Neuroderm, Intec Pharma, Teva, Genzyme-Sanofi, Biogen, Lysosomal Therapeutics, Denali, Cellanis, GaitBetter, Vibrant and Sionara; that he holds shares 
or options in Lysosomal Therapeutics, Cellanis, GaitBetter and Vibrant; that he has received royalties from Lysosomal Therapeutics; that he received honorarium from UCB, Teva, Novartis, Abbvie, GenzymeSanofie, Neuroderm, Bial, Shire, MDS; that he have chaired the DSMBs for Teva and Pharma2B; that he is a PI on a Center Grant given by Biogen to TLVMC; that he has submitted a patent application on the use of body-fixed sensors for assessing PD symptoms, the intellectual property rights for which are held by the Tel Aviv Medical Center, and that he received grants from Teva, Biogen, LTI, ISF, EU, NIH, MJFF, Parkinson Foundation, and Pfizer.

Anat Mirelman serves as chair of the Michael J Fox Foundation task force on gait. She has submitted a patent application on the use of bodyfixed sensors for assessing PD symptoms, the intellectual property rights for which are held by the Tel Aviv Medical Center.

Jeffrey Hausdorff serves on the Movement Disorders Society Technology Task Force and on Michael J Fox Foundation task force on gait, and on advisory boards for Sanofi and Biogen. He has submitted a patent application on the use of body-fixed sensors for assessing PD symptoms, the intellectual property rights for which are held by the Tel Aviv Medical Center.

\section{Acknowledgements}

We thank the study participants and all of those who contributed to the V-TIME project. This work was funded in part by a grant from the European Commission.

\section{Appendix A. Supplementary data}

Supplementary data to this article can be found online at https:// doi.org/10.1016/j.parkreldis.2019.01.022.

\section{References}

[1] S. Del Din, A. Godfrey, B. Galna, S. Lord, L. Rochester, Free-living gait characteristics in ageing and Parkinson's disease: impact of environment and ambulatory bout length, J. NeuroEng. Rehabil. 13 (2016) 46.

[2] R.C. van Lummel, S. Walgaard, M. Pijnappels, P.J. Elders, J. Garcia-Aymerich, J.H. van Dieen, et al., Physical performance and physical activity in older adults: associated but separate domains of physical function in old age, PLoS One 10 (2015) e0144048.

[3] E. Giannouli, O. Bock, S. Mellone, W. Zijlstra, Mobility in old age: capacity is not performance, BioMed Res. Int. 2016 (2016) 3261567.

[4] P. Tamburini, F. Storm, C. Buckley, M.C. Bisi, R. Stagni, C. Mazza, Moving from laboratory to real life conditions: influence on the assessment of variability and stability of gait, Gait Posture 59 (2018) 248-252.

[5] E.A.F. Ihlen, A. Weiss, Y. Beck, J.L. Helbostad, J.M. Hausdorff, A comparison study of local dynamic stability measures of daily life walking in older adult communitydwelling fallers and non-fallers, J. Biomech. 49 (2016) 1498-1503.

[6] S.M. Rispens, K.S. van Schooten, M. Pijnappels, A. Daffertshofer, P.J. Beek, J.H. van Dieen, Identification of fall risk predictors in daily life measurements: gait characteristics' reliability and association with self-reported fall history, Neurorehabilitation Neural Repair 29 (2015) 54-61.

[7] J.M.T. van Uem, B. Cerff, M. Kampmeyer, J. Prinzen, M. Zuidema, M.A. Hobert, et al., The association between objectively measured physical activity, depression, cognition, and health-related quality of life in Parkinson's disease, Park. Relat. Disord. 48 (2018) 74-81.

[8] A. Sanchez-Ferro, W. Maetzler, Advances in sensor and wearable technologies for Parkinson's disease, Mov. Disord. 31 (2016) 1257.
[9] A. Weiss, T. Herman, N. Giladi, J.M. Hausdorff, Objective assessment of fall risk in Parkinson's disease using a body-fixed sensor worn for 3 days, PLoS One 9 (2014) e96675.

[10] S. Del Din, A. Godfrey, C. Mazza, S. Lord, L. Rochester, Free-living monitoring of Parkinson's disease: lessons from the field, Mov. Disord. 31 (2016) 1293-1313.

[11] S. Del Din, B. Galna, A. Godfrey, E.M. Bekkers, E. Pelosin, F. Nieuwhof, et al. Analysis of free-living gait in older adults with and without Parkinson's disease and with and without a history of falls: identifying generic and disease specific characteristics, J Gerontol A Biol Sci Med Sci (2017 Dec 30), https://doi.org/10.1093/ gerona/glx254 [Epub ahead of print].

[12] D. Johansson, K. Malmgren, M.M. Alt, Wearable sensors for clinical applications in epilepsy, Parkinson's disease, and stroke: a mixed-methods systematic review, J. Neurol. 265 (2018) 1740-1752.

[13] M. El-Gohary, S. Pearson, J. McNames, M. Mancini, F. Horak, S. Mellone, et al., Continuous monitoring of turning in patients with movement disability, Sensors 14 (2013) 356-369.

[14] C.G. Goetz, S. Fahn, P. Martinez-Martin, W. Poewe, C. Sampaio, G.T. Stebbins, et al., Movement disorder society-sponsored revision of the unified Parkinson's disease rating Scale (MDS-UPDRS): process, format, and clinimetric testing plan, Mov. Disord. 22 (2007) 41-47.

[15] J.M. Hausdorff, Gait dynamics, fractals and falls: finding meaning in the stride-tostride fluctuations of human walking, Hum. Mov. Sci. 26 (2007) 555-589.

[16] M.L. Dontje, M.H. de Greef, A.D. Speelman, N.M. van, W.P. Krijnen, R.P. Stolk, et al., Quantifying daily physical activity and determinants in sedentary patients with Parkinson's disease, Park. Relat. Disord. 19 (2013) 878-882.

[17] M.E. Cusso, K.J. Donald, T.K. Khoo, The impact of physical activity on non-motor symptoms in Parkinson's disease: a systematic review, Front. Med. 3 (2016) 35.

[18] M. Lauze, J.F. Daneault, C. Duval, The effects of physical activity in Parkinson's disease: a review, J. Parkinson's Dis. 6 (2016) 685-698.

[19] H. Terashi, H. Utsumi, Y. Ishimura, H. Mitoma, Independent regulation of the cycle and acceleration in parkinsonian gait analyzed by a long-term daily monitoring system, Eur. Neurol. 69 (2013) 134-141.

[20] A. Rodriguez-Molinero, A. Sama, C. Perez-Lopez, D. Rodriguez-Martin, S. Alcaine, B. Mestre, et al., Analysis of correlation between an accelerometer-based algorithm for detecting parkinsonian gait and UPDRS subscales, Front. Neurol. 8 (2017) 431.

[21] A.L. Silva de Lima, L.J.W. Evers, T. Hahn, N.M. de Vries, M. Daeschler, B. Boroojerdi, et al., Impact of motor fluctuations on real-life gait in Parkinson's patients, Gait Posture 62 (2018) 388-394.

[22] A.S. Buchman, P.A. Boyle, L. Yu, R.C. Shah, R.S. Wilson, D.A. Bennett, Total daily physical activity and the risk of AD and cognitive decline in older adults, Neurology 78 (2012) 1323-1329.

[23] M. Reiner, C. Niermann, D. Jekauc, A. Woll, Long-term health benefits of physical activity - a systematic review of longitudinal studies, BMC Public Health 13 (2013) 813.

[24] A. Mirelman, L. Rochester, I. Maidan, D.S. Del, L. Alcock, F. Nieuwhof, et al., Addition of a non-immersive virtual reality component to treadmill training to reduce fall risk in older adults (V-TIME): a randomised controlled trial, Lancet 388 (2016) 1170-1182.

[25] A. Mirelman, L. Rochester, M. Reelick, F. Nieuwhof, E. Pelosin, G. Abbruzzese, et al., V-TIME: a treadmill training program augmented by virtual reality to decrease fall risk in older adults: study design of a randomized controlled trial, BMC Neurol. 13 (2013) 15

[26] S.A. Combs, M.D. Diehl, J. Filip, E. Long, Short-distance walking speed tests in people with Parkinson disease: reliability, responsiveness, and validity, Gait Posture 39 (2014) 784-788.

[27] A. Doherty, D. Jackson, N. Hammerla, T. Plotz, P. Olivier, M.H. Granat, et al., Large Scale population assessment of physical activity using wrist worn accelerometers: the UK biobank study, PLoS One 12 (2017) e0169649.

[28] R.J. Dawe, S.E. Leurgans, J. Yang, J.M. Bennett, J.M. Hausdorff, A.S. Lim, et al., Association between quantitative gait and balance measures and total daily physical activity in community-dwelling older adults, J Gerontol A Biol Sci Med Sci 73 (2018) 636-642.

[29] H. Besson, U. Ekelund, S. Brage, R. Luben, S. Bingham, K.T. Khaw, et al., Relationship between subdomains of total physical activity and mortality, Med. Sci. Sports Exerc. 40 (2008) 1909-1915.

[30] L. Rochester, S.F. Chastin, S. Lord, K. Baker, D.J. Burn, Understanding the impact of deep brain stimulation on ambulatory activity in advanced Parkinson's disease, J. Neurol. 259 (2012) 1081-1086. 